\title{
HISTO RIA
}

\section{E ORIGEN 円NOMENOLÓGCO DE “CUIDADO” Y LA IMPORTANCIA DE CONCEPTO DE TIEMPO EN LA HISTORIA DE LA ENFRMERÍA} José Siles González; Carmen Solano Ruiz Profesores del Departamento de Enfermería. Universidad de Alicante

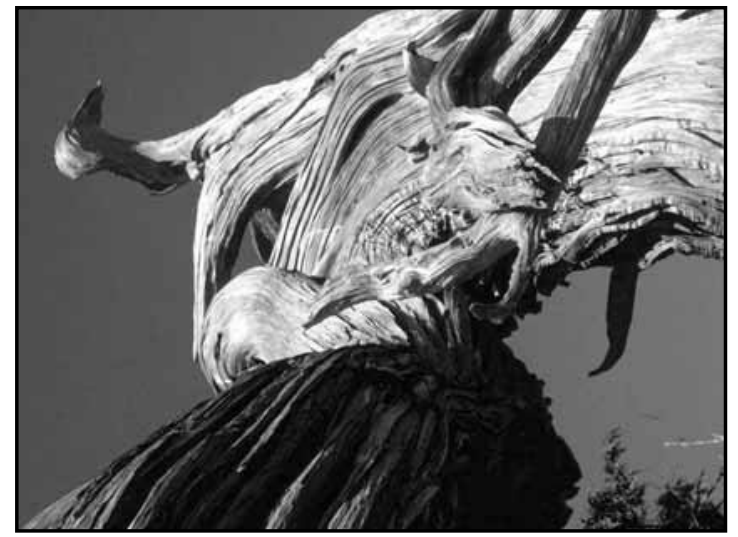

PHENOMENOLOGICAL ORIGIN OF "CARE" AND IMPORTANCE OF THE CONCEPT OF TIME IN NURSING HISTORY

\section{SUMMARY}

$\mathrm{T}$ The general aim of this work consists, fundamentally, in meditate on the time concept in the cares context, facilitating the understanding of the phenomenological nursing nature, and also, to analyze historically the phenomenological origin of the concepts of "care" and "cure" identifying its presence and meaning in the myths. Considering that the phenomenological nature of cares is described and explained in oral traditions integrated in myths and fables, constitutes the hypothesis of this study. Results: Heidegger employs narrative materials as the fable to develop the concepts of care and cure and integrate them in its henomenological work. The care is a concept whose great epistemologic potential can contribute to the process of consolidation of the nursing discipline. Conclusions: Heidegger, in "Being and
Time", develop the phenomenological concept of "care" and "cure" in a narrative source like the fable of Higinio (S. I a.C) "Fable of the man and the world", Time constitutes the existential riverbed for which there pass the diverse historical moments or "Kairós" that the human being (at individual, family or community level) dedicates to the satisfaction of each and every of his needs.

Keywords: Nursing history, phenomenology of cares and culture care.

\section{RESUMEN}

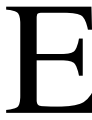

1 objetivo general de este trabajo consiste, fundamentalmente, en reflexionar sobre el concepto de tiempo en el contexto de los cuidados facilitando la comprensión de la naturaleza fenomenológica de la enfermería, para lo que resulta ineluctable la consideración de un propósito preliminar: analizar históricamente el origen fenomenológico de los conceptos de "cuidado" y "cura" identificando su presencia y significado en los mitos. Considerar que la naturaleza fenomenológica de los cuidados se describe y explica en tradiciones orales que dan lugar a los mitos y las fábulas constituye la hipótesis de este estudio. Resultados: Heidegger emplea materiales narrativos como la fábula para desarrollar los conceptos de cuidado y cura e integrarlos en su obra fenomenológica. El cuidado es un concepto cuyo gran potencial epistemológico puede contribuir al proceso de vertebración de la disciplina enfermera.

Conclusiones: Heidegger, en "El ser y el tiempo", establece el concepto fenomenológico de cuidado y el de cura. Una fuente narrativa como la fábula 
de Higinio (S. I a.C) "Fábula del hombre y el mundo", constituye un referente esencial para entender la importancia filosófica, fenomenológica y epistemológica del cuidado. El tiempo constituye el cauce existencial por el que transcurren los diversos momentos históricos o "Kairós" que el ser humano (a nivel individual, familiar o comunitario) dedica a la satisfacción de todas y cada una de sus necesidades.

Palabras clave: historia de la enfermería, fenomenología de los cuidados, cultura de los cuidados.

\section{I.- INTRODUCCIÓN}

El objetivo general de este trabajo consiste, fundamentalmente, en reflexionar sobre el concepto de tiempo en el contexto de los cuidados facilitando la comprensión de la naturaleza fenomenológica de la enfermería, para lo que resulta ineluctable la consideración de un propósito preliminar: analizar históricamente el origen fenomenológico de los conceptos de "cuidado" y "cura" identificando su presencia y significado en los mitos. Para vertebrar el proceso de reflexión se ha diseñado una batería de objetivos específicos:

- Demostrar la equivalencia etimológica de los conceptos "cura" y "cuidado".

- Identificar y analizar las fuentes narrativas y poéticas empleadas por Heidegger para fundamentar su obra en relación a los cuidados.

- Explicar el papel de "Cura" como dispensadora "preontológica" del cuidado y fundamentadora de la vida del hombre (dar a luz al hombre).

- Describir la ética del cuidado (desde la perspectiva fenomenológica)

- Demostrar, en consecuencia, el enorme potencial semántico del término "cuidado" como instrumento de desarrollo espistemológico de la disciplina enfermera.

Se ha partido de las siguientes hipótesis:

- La naturaleza fenomenológica de los cuidados se describe y explica en tradiciones orales que dan lugar a los mitos y las fábulas.

- Heidegger emplea materiales narrativos como la fábula para desarrollar los conceptos de cuidado y cura e integrarlos en su obra fenomenológica.
- Identificar y comprender la ética como una forma esencial del "cuidado" del ser humano.

- El cuidado es un concepto cuyo gran potencial epistemológico pude contribuir al proceso de vertebración de la disciplina enfermera.

- El ritmo y el estilo de vida están determinados por la forma de interpretar el tiempo y el cuidado del ser humano requiere un tiempo (Kairós) para la satisfacción de sus necesidades

\section{II.- ESTADO DE LA CUESTIÓN}

A lo largo de la historia, distintos autores han aportado sus particulares interpretaciones del tiempo. Tal vez sea Platón quien se ha referido al tiempo de forma más estética: "imagen móvil de la eternidad". O la perplejidad de Heráclito observando cómo discurre el agua convirtiendo en irrepetible aquel momento, pero tal vez sin percatarse que él mismo discurre -su existencia- como el agua y que, al igual que el río, jamás volverá a ser el mismo de aquel preciso instante. Nada ni nadie se escapa al efecto del tiempo, aunque existe una mayor complejidad para entenderlo si nos alejamos del tiempo puramente biológico o vital: nacimiento, crecimiento y desarrollo hasta la edad adulta, envejecimiento progresivo hasta la ancianidad y, final e irremediablemente, muerte. Han sido varios los autores integrados en diferentes tendencias que han reflexionado sobre la naturaleza del tiempo y su incidencia en la vida cotidiana y en la historia (Bloch, 1999; Braudel, 2002; Bueno, 1978; Burke, 2003; Febvre, 1999) considerando siempre que el objeto de la historia es el fenómeno humano a través de ese complejo "constructo" o "convención" que es el tiempo.

Conforme nos alejamos de la interpretación biológica o psicológica (esperar algo es alargar el tiempo) del tiempo resulta más complejo entenderlo: el tiempo geológico (millones de años de evolución de la litosfera, la hidrosfera, etc.), el tiempo físico, el tiempo histórico; pero sobre todo, es el tiempo cosmológico (con la posibilidad irresoluta de infinitud) el que resulta más incomprensible para le mente humana (Whiltrow, 1990). En consecuencia, para comprender la relación entre tiempo y acontecimiento es aconsejable clarificar el término Kairós, concepto que acuñaron los griegos planteando desde la antigüedad la interpretación del ins- 
tante, el momento, como algo más que mero presente, intentando demostrar que el presente no era únicamente un puente entre el pasado y el futuro cuyo paso por la historia era tan fugaz como irrelevante (Gracia, 1998). Los filósofos griegos emplearon el término "Crhonos" para referirse al tiempo cronológico y el concepto "Kairós" para identificar la importancia del momento vivido oportunamente, del tiempo que requiere cada cosa: tiempo de incubación, tiempo de convalecencia, tiempo de crisis, tiempo de epidemia, tiempo de cuidados, tiempo de tratamiento, tiempo de vacas flacas o gordas, tiempo de amar, tiempo de morir, tiempo de nacer, tiempo de fecundación, etc. (Siles, 1999). El origen fenomenológico del cuidado desde la perspectiva epistemológica y hermenéutica fue abordado por diferentes autores considerando la integración de los cuidados en la vida cotidiana (Siles, 1997), y las variantes interpretativas en el proceso de satisfacción de necesidades según las diferentes culturas (Annels, 1996). La relación entre enfermería y fenomenología se ha empleado también para reflexionar sobre el proceso de construcción social de la enfermería (Siles, 2004) y para valorar la dialéctica construccionista-deconstruccionista en todos los aspectos que tienen que ver con la incidencia del género en enfermería (Siles, 2005). La importancia de los enfoques fenomenológicos en cuestiones de la práctica enfermera tiene numerosos ejemplos en muy diferentes campos: en la aparición del fenómeno de la fatiga en procesos crónicos y tan estresantes como el cáncer (Pearce \& Richardson, 1996); o en el estudio de las vivencias de los pacientes coronarios en las unidades de cuidados críticos (Solano y Siles, 2005). El análisis específico de la relación entre enfermería y fenomenología fue abordado por autores desde la perspectiva de su relación con la obra de Husserl (Paley, 1997), o poniendo el énfasis analítico en la relación entre el carácter holístico de la enfermería y la fenomenología (Bishop \& Scudder, 1997).

\section{III.-FUENTES Y MÉTODOS}

Para vertebrar el proceso de reflexión sobre el tiempo y la historia se ha empleado el método histórico desde la perspectiva de la historia estructural (Vilar, 2001) siguiendo los criterios aportados por Aróstegui (2001) tanto para el proceso de bús- queda heurística como para el análisis de los textos objeto de estudio. El proceso hermenéutico desarrollado para analizar la naturaleza fenomenológica del "cuidado" y la "cura" se ha aplicado a la fábula de Higinio: "El hombre y el Mundo" (siglo I a.C.) y se ha realizado el pertinente análisis comparativo al texto de Heidegger "El ser y el tiempo" en el que trata los conceptos de "cuidado" y "cura" desde la perspectiva de su teoría fenomenológica.

\section{IV.-DESARROLLO DEL TEMA}

LAS ADAPTACIONES DEL MÉTODO FENOMENOLÓGICO A LA HISTORIA DE LA ENFERMERÍA: DOS FORMAS COMPLEMENTARIAS Y DIALÉCTICAS DE ESTUDIAR EL COMPLEJO MUNDO DE LOS CUIDADOS.

El estudio de la evolución de los cuidados a través del tiempo puede llegar a complementarse mediante la adaptación de las perspectivas fenomenológicas a la historia de la enfermería. El concepto de "fenomenología, etimológicamente, se deriva del griego "phainomenon" (todo lo que se muestra, manifiesta o aparece ante un individuo que se interroga) y "logos" (que significa estudio o tratado). Para Husserl, un hombre formado en las matemáticas y disconforme con los métodos humanísticos de interpretación de la realidad, la fenomenología es el estudio del fenómeno en sí mismo. En consecuencia, el significado etimológico de fenomenología es el tratado de lo que se manifiesta o de lo aparente. Empleando como herramientas históricas las dos corrientes fenomenológicas más difundidas (las aportadas por Husserl y Heidegger, respectivamente) se desvelan dos formas complementarias de interpretar el complejo mundo de los cuidados a través del tiempo:

- Los cuidados como concepto universal, esencial $\mathrm{y}$, por tanto, común para todas las culturas en todas las épocas históricas e independientemente de las ideologías, creencias, y presupuestos científicos: el cuidado como concepto y un fenómeno dado en sí mismo a la conciencia (según el proceso reduccionista de Husserl, 1994). Lo esencial de los cuidados hace referencia a sus características, propiedades y relaciones más profundas y estables, permitiendo indagar históricamente tanto en sus antecedentes como en las tendencias que han marcado su evolución a través del tiempo. 
- Los cuidados como concepto y fenómeno cuyos significados y prácticas están influidos por el contexto social, político, ideológico, religioso y científico en el que tiene lugar (tal como postula Heidegger, 2000). En este tipo de interpretación, el cuidado constituye un fenómeno que está compuesto por un conjunto de propiedades y relaciones del objeto diversas, externas, dinámicas, percibidas directamente a través de los sentidos, y, en definitiva, se puede estudiar más el modo que tiene la esencia del cuidado para manifestarse o revelarse que lo esencial del cuidado.

En el primer caso, siguiendo a Husserl, se profundiza en lo esencial de los fenómenos, lo que se correspondería con una visión estructural de la historia de los cuidados, dado que la historia estructural bucea en los cimientos de las sociedades para explicar la influencia de factores que "no se aprecian" o "no aparecen" en la superficie de los fenómenos. Esta visión fenomenológica y estructural de la historia de la enfermería permite el estudio en profundidad de los cuidados revelando sus características esenciales que son compartidas por diferentes culturas en distintas fases históricas. Para Platón la esencia constituye el ideal de las "ideas" (la enfermedad existe en la medida que tengo una idea de lo que es la enfermedad); para Hegel lo esencial es "la idea absoluta"; Kant declara su agnosticismo al reconocer la incapacidad de conocer la esencia objetiva de las cosas (dado que todos los fenómenos se perciben subjetivamente); otros, como Dewey, no distinguen entre esencia y fenómeno, dado que piensan que es la misma cosa; y, por último, también los hay partidarios de identificar el fenómeno con la sensación negando por completo la esencia (fenomenalismo de Mach).

La gran ventaja la constituye la universalidad, dado que hablamos de todo lo que tiene de esencial el cuidado $\mathrm{y}$, en consecuencia, es entendido en todas las épocas y culturas como una misma cosa que tiene las mismas características y finalidades. Sin embargo, también se paga un precio: esa visión esencial no se ajusta del todo (si se valoran las creencias, experiencias subjetivas, ideologías, etc.) a ninguna cultura en ninguna fase histórica. Se trata, pues, de valorar la esencialidad y universalidad de los cuidados.

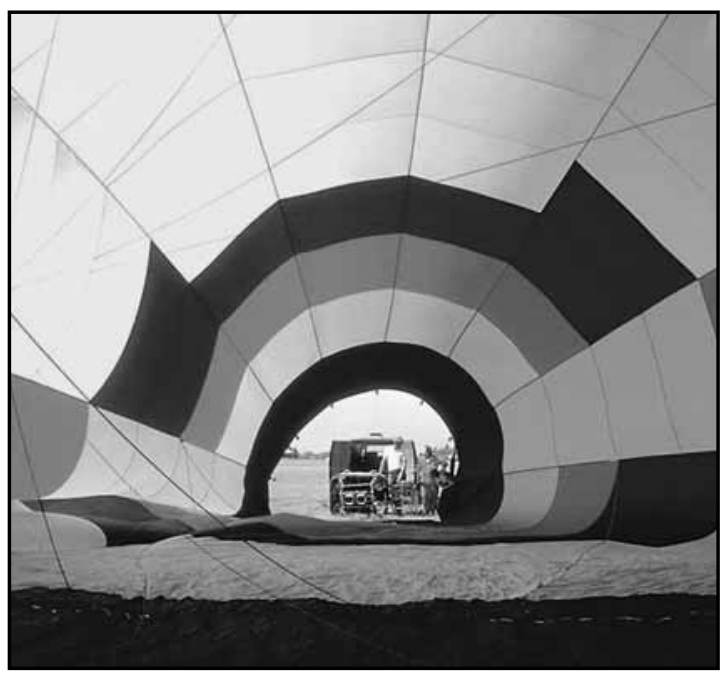

Por otro lado, mediante la aplicación de la fenomenología heideggeriana a la historia de la enfermería se obtiene una visión coyuntural permitiendo la identificación y comprensión de los fenómenos implicados en cuidados de salud en un contexto específico en el que se consideran las creencias, las experiencias subjetivas, las ideologías, etc. Se trata aquí de valorar la influencia que las creencias, el bagaje experiencial y el entorno mismo, tienen en la interpretación de los significados que se dan en las situaciones vida-salud-enfermedad-muerte.

De forma que, mediante la aplicación de estas dos perspectivas fenomenológicas a la historia de la enfermería, se pueden realizar estudios estructurales y coyunturales de los cuidados de salud en las diferentes épocas sin perder la tensión dialéctica entre la incidencia que los aspectos esenciales, por un lado, y los factores culturales, religiosos e ideológicos, por otro, tienen en el complejo resultado final: los cuidados.

Desde esta perspectiva integradora de los aspectos esenciales y subjetivos de los cuidados, se aporta una definición que intenta subsanar el defecto inherente a toda definición con vocación sentenciadora: la mutilación de lo definido (reduccionismo):

"El producto de la reflexión sobre las ideas, hechos y circunstancias (reflexión teórica, práctica y situacional) relacionadas con el proceso optimizador de las necesidades que garantizan la satis- 
facción integral de todas y cada una de las etapas que constituyen la vida humana desde el momento del nacimiento hasta la muerte" (Siles, 2000).

\section{El ORIGEN FENOMENOLÓGICO DE LOS TÉRMINOS "CURA" Y "CUIDADO"}

Toda la historia del hombre, entendida esta como el paso por la vida, es la historia de una preocupación continua por mantener unos niveles de salud de acuerdo con la interpretación que en cada cultura se le da al concepto "salud". Leyendo a Heidegger, especialmente su obra "El ser y el tiempo", se puede llegar a establecer el concepto fenomenológico de cuidado y el de cura.

Paradójicamente, la enfermería, aun estando omnipresente ante el dolor, la angustia y la muerte, se ha mantenido cautelosamente alejada de la reflexión vivencial y fenomenológica del cuidado.

La cuestión es, ¿qué es lo que Heidegger percibió o no percibió, lo que retuvo y lo que buscó cambiar de ese período que abarca los dos primeros tercios del siglo $\mathrm{XX}$ ?. Indudablemente Heidegger no fue el protagonista de su época, en cualquier caso no de su época histórica y política, pues lo fueron Hitler y, a su manera, también Lenin y Stalin; pero tampoco paso sin pena ni gloria por los importantes momentos históricos que le tocaron vivir. En un momento histórico en el que había que tener "especial cuidado" o aplicar el concepto de "cuidado" en el más amplio sentido del término, logró evitar su participación como soldado en las dos primeras guerras mundiales y, además, tuvo un importante empleo que le permitió vivir con relativa soltura dado los tiempos que corrían.

\section{ETIMOLOGÍA Y SIGNIFICADO FENOMENO- LÓGICO DEL CONCEPTO DE CURA EN HEI- DEGGER}

El concepto de cura deriva del latín y es tanto el sustantivo (cura, curae) como el imperativo del verbo curare (cura; curate). Todo el grupo léxico ha mantenido los significados de cuidar y cuidado. Empleado en especial en medicina adopta otro significado (cura, curación, curandero) perdiéndose casi del todo el significado original de cuidado genérico. Cura es, además, una de las piezas de un nombre más largo: párroco con cura de almas, que se redujo al de cura párroco. Por otro lado las
Sinecuras, es decir el oficio de párroco sin obligación de cuidar de las almas. Etimológicamente, la palabra "seguridad" proviene del latín sine cura (sin cuidado, sin preocupación). Hay momentos de nuestra vida en los que nos gustaría desenvolvernos más despreocupadamente de lo que normalmente podemos permitirnos respecto a (Rodríguez, 2005): salud propia y de familia y amigos; puesto de trabajo; aditivos de alimentos; callejear por las noches por cualquier lugar sin preocupación, etc.

La utilización de las palabras cura y cuidado es, en Heidegger, integrativa y es del todo correcta, dado que emplea el mismo término para referirse al cuidado y a la cura (sorge).

Sin embargo diferencia entre "cura" como nombre propio femenino que es la dispensadora de cuidados durante toda la vida del ser (nacimiento hasta su muerte). En El ser y el tiempo (1927) describe lo que llamaba la "estructura de la cotidianidad", o 'ser en el mundo', que era una interrelación de acciones, actitudes, conocimientos, intenciones, etc. (enfoque holístico).

\section{LA INFLUENCIA DE LAS FUENTES NARRA- TIVAS EN LA INTEGRACIÓN EN LA FENO- MENOLOGÍA DE LOS CONCEPTOS DE CUI- DADO Y CURA.}

La influencia de la narrativa y la poesía en "El ser y el tiempo" (1927) se aprecia por las referencias a Nietzsche, Dostoievski, Hölderlin, Rilke y otros muchos autores. Aunque, como tantos otros, se afilió al partido nacional socialista y llegó a ser Rector de la universidad de Friburgo en época nazi, Heidegger estuvo siempre preocupado por una cuestión esencial para él: la condición efímera e inestable del ser humano desde el momento de su nacimiento al de su muerte. Reflexionó sobre los conceptos de cura y cuidado llegando a integrarlos en su teoría fenomenológica. Para ello se ayudo de materiales narrativos leyendo autores como los anteriormente citados especialmente preocupados por la razón y finalidad de la existencia. Heidegger pensaba que el individuo $\mathrm{y}$, por extensión el ser humano, es lo que uno hace en el mundo (Dasein), $y$ en todo lo que uno hace en el mundo -especialmente en el proceso de satisfacción de sus necesidades- aplicar el cuidado suficiente. La salud -tanto la mental como la física y social- está direc- 
tamente relacionada con el nivel de cuidado integrado en el mecanismo general de satisfacción de necesidades del ser humano. En definitiva para Heidegger el cuidado es interpretado como la estructura fundamental del ser y la cotidianidad.

\section{El cuidado como estructura fundamental del ser y la cotidianidad}

La dialéctica entre estilo de vida y nivel saludenfermedad esta directamente relacionado con el concepto de cuidado. El cuidado es, también, una forma de llegar a alcanzar cierto control sobre nuestras vidas, nuestro destino, y esto es para Heidegger el ser o no ser propietario de la existencia. Heidegger estaba especialmente preocupado por la impropiedad, dado que pensaba que la impropiedad es la forma originaria de nuestro ser en el mundo (previa al nacimiento y posterior a la muerte). La impropiedad es un existenciario, es decir, una estructura fundamental del ser que lo envuelve desde el primer momento, y lo acompañará el resto de la vida (el cuidado funciona como antídoto). Esta sensación de impropiedad provoca en el hombre desamparo e indefensión ante el dolor, la enfermedad y la muerte. Sólo mediante el cuidado logra el hombre fijarse en lo que le conviene desde el punto de vista de la satisfacción de sus necesidades, en general, y de la salud, en particular.

CUIDADO: Mantener la atención, fijarse, estar en lo que se está haciendo. Procurarse un sitio en el mundo. Andarse con cautela, No perder detalle (importante) ni bajar (descuidadamente) la guardia. El cuidado es estar dispuesto e instantáneamente disponible. Siempre listo, siempre despierto.

\section{EL ORIGEN DEL HOMBRE Y EL CUIDADO EN HEIDEGGER}

Para analizar el origen del hombre y desarrollar los conceptos de cura y cuidado, Heidegger emplea, de nuevo, las fuentes narrativas, concretamente una fábula escrita por Higinio en la primera mitad del siglo I a.C: "Fábula del hombre y el mundo"

Una vez llegó Cura a un río y vio terrones de arcilla. Cavilando, cogió un trozo y empezó a modelarlo. Mientras piensa para sí qué había hecho, se acerca Júpiter. Cura le pide que infunda espíritu al modelado trozo de arcilla. Júpiter se lo concede con gusto. Pero al querer Cura poner su nombre a su obra, Júpiter se lo prohibió, diciendo que debía dársele el suyo. Mientras Cura y Júpiter litigaban sobre el nombre, se levantó la Tierra y pidió que se le pusiera a la obra su nombre, puesto que ella era quien había dado para la misma un trozo de su cuerpo. Los litigantes escogieron por juez a Saturno. Y Saturno les dio la siguiente sentencia evidentemente justa:

Tú, Júpiter, por haber puesto el espíritu, lo recibirás a su muerte; tú, Tierra, por haber ofrecido el cuerpo, recibirás el cuerpo. Pero por haber sido Cura quien primero dio forma a este ser, que mientras viva lo posea Cura. Y en cuanto al litigio sobre el nombre, que se llame homo, puesto que está hecho de humus (tierra).

Higinio "Fabula del hombre y el mundo" (4030 a.C.)

En esta fábula la "cura" (cuidado) aparece en conexión con la conocida concepción del hombre como: Compuesto de cuerpo (tierra) y espíritu (holístico). El hombre tiene el "origen" de su ser en la cura. Y una vez vivo es retenido, dominado por el cuidado mientras esta en el Mundo. El hombre está en el mundo (ser en el mundo) mediante la acción de la "cura"que le dota de entidad: su nombre "homo" no lo recibe de su propio ser, sino de aquello de lo que está hecho "humus". Para Heidegger la "cura" tiene un doble significado: "hingable" (entrega) y "sorgen" (cuidado). La cura, pues, aparece como la figura femenina que, metafóricamente, hace posible el parto del hombre $y$, tras la existencia de éste, se transforma en su cuidadora. La cura es la entidad "femenina" que modela al ser mediante arcilla (tierra) y la mediadora que le facilita el espíritu (Júpiter) acompañándolo hasta su muerte en forma de cuidados. La utilización de las palabras cura y cuidado es en Heidegger, desde la perspectiva etimológica, es más correcta que la utilización disociativa que en nuestros días es la más difundida en medicina y enfermería. De todo lo anterior se deduce que el concepto "cuidado" tiene un enorme potencial semántico que debe ser empleado para desarrollar de forma epistemológica la disciplina enfermera, dado que, en definitiva, el cuidado acompaña al 
hombre desde su nacimiento hasta su muerte $\mathrm{y}$ constituye, desde la perspectiva fenomenológica, la estructura fundamental de su existencia o "existenciario"

\section{ÉTICA DEL CUIDADO}

El ser humano en sus acciones ha de poner cuidado para acompañar su existencia de la dignidad y la ética necesarias. La finalidad y justificación del cuidado es "velar" para que el ser desarrolle todas sus potencialidades para que llegue a ser él mismo, el realmente es en un contexto históricocultural, el hombre en su plenitud a nivel holístico: biopsicosocial.

\section{"Hay que apreciar cuando hoy un hombre tiene todavía la aspiración de ser algo entero"}

(Robert. Musil)

Nozick (1988) señala que para el desarrollo ético del cuidado (llegar a ser con dignidad/ ética) hay que mantener los niveles adecuados de: consistencia/coherencia, cuidado y consecuencias.

\section{Consistencia/coherencia}

Se interioriza la conducta para modelar las acciones intencionalmente en base a unos determinados valores (sociales, de salud, ideología, religión, etc.)

\section{Cuidado}

Reflexión, atención sobre una amplia red de relaciones con el fin de mantener el equilibrio entre el pensamiento, los valores y la conducta de forma holística.

\section{Consecuencias}

El aspecto objetivo de la ética son los resultados prácticos derivados de las conductas/ praxis.

Un ejemplo de cuidado desde la perspectiva ética la constituye la propia vida de el autor de "El ser y el tiempo: resulta evidente que Heidegger se ve sometido a la presión de su época como ser arrojado a un espacio y un tiempo en el que afloran las contradicciones (nazismo, racismo, etc.); pero también es cierto que se cuida de dejarse arrastrar del todo (aunque no puede permanecer completa- mente aislado del fenómeno histórico). Heidegger, que interpreta el cuidado como la estructura fundamental del ser, mantiene la consistencia/coherencia que fundamenta en sus valores y, mediante la reflexión crítica, intenta mantenerse dentro de la dignidad personal ante las enormes presiones ambientales. Los resultados prácticos de esta ética del cuidado se manifiestan, tienen como consecuencia, la visión integral y relacional de factores como: salud, la ideología, los valores y la capacidad de reflexión que permite mantener la dosis básica de libertad interpretativa para que pueda hablarse de una existencia vivida en la dignidad. Dicho de otro modo, desde esta perspectiva, se puede apreciar la salud en sus diferentes planos: social, fisiológico y mental.

Cuando el viento, saltando brusco, gruñe entre la armazón de la cabaña, ya el día se pone ceñudo...

\section{Nunca llegamos a pensamientos. Llegan ellos a nosotros. Tal es la hora propicia al diálogo.} (Heidegger)

\section{RELACIÓN KAIRÓS-SATISFACCIÓN DE NECESIDADES HUMANAS}

El momento, el instante es el lugar donde se escenifica la vida cotidiana y donde se va configurando la personalidad y las condiciones físicas: momento a momento, instante a instante, el hombre, mediante la ejecución rutinaria de esos hábitos tan relacionados con la satisfacción de necesidades, puede favorecer el desarrollo de una determinada enfermedad o, por el contrario, potencia una forma determinada de satisfacer sus necesidades que no sólo no es contraria con el mantenimiento de una vida equilibrada y sana, sino que resulta imprescindible para el mantenimiento de ésta. A largo de la historia los hombres han estado inmersos en sociedades que les han obligado a llevar diferentes "ritmos de vida". Esos ritmos de vida han estado formados por diferentes tiempos: Desde los tiempos de recolección salvaje y caza en las sociedades primitivas, pasando por el tiempo de siembra y cosecha desde las primeras sociedades neolíticas, hasta el tiempo de guerra de los períodos más álgidos de las culturas antiguas y medie- 
vales, que eran seguidos por breves episodios de descanso bélico (tiempo de paz); sin embargo, en ninguna época histórica el hombre ha estado tan desorientado con respecto a la organización temporal de sus actividades como lo está a finales del siglo XX. Los cambios horarios y la generación incesante de nuevas necesidades proyectadas desde los medios de comunicación han provocado la desvinculación entre la naturaleza y el tiempo contribuyendo a un consumismo desordenado en el que la afirmación: "un tiempo para cada cosa" se ha convertido en una expresión vacía de contenido para una humanidad que podría pasarse las veinticuatro horas del día delante de un televisor. Desde luego, en este contexto, es preciso poner orden. Sin duda sigue existiendo un Kairós para satisfacer cada una de las necesidades humanas y en un contexto social tan agobiante como el impuesto por este fin de siglo se hace preciso que los individuos, las familias y comunidades, involucren un tiempo idóneo para la satisfacción adecuada de cada una de sus necesidades como primer requisito para alcanzar el éxito en dicho proceso (TABLA I.5).

\section{TABLA I.5}

\section{SATISFACCIÓN DE NECESIDADES} Y KAIRÓS

Kairós-patrón respiratorio

Kairós-comer/beber

Kairós-patrón de eliminación

Kairós-movimiento-mantenimiento

Kairós-dormir-descansar

Kairós-vestido-abrigo adecuado.

Kairós-mantenimiento temperatura.

Kairós-aseo-higiene personal.

Kairós-evitar peligros ambientales.

Kairós-comunicación.

Kairós-religión.

Kairós-ocupación-empleo

Kairós-lúdico

Kairós-aprendizaje-descubrimiento.

Kairós-patrón sexual

Fuente: Elaboración propia basado en Henderson (1991)

\section{EL KAIRÓS COMO MENSAJE HISTÓRICO}

El instante va más allá del mero presente, porque puede erigirse en el mensajero de un asunto tan crucial y revelador que su influencia no va a extinguirse con el presente, sino que va a ir mucho más allá. Desde el plano subjetivo, el diagnóstico de una enfermedad no se agota en el momento de la comunicación, sino que constituye todo un fenómeno que va a proyectarse durante buena parte de la vida del sujeto y, en algunos casos, que va a acompañarle durante el resto de su existencia. Es tal la densidad de ese instante que genera tiempo de duda e incertidumbre, donde se agolpan los sentimientos, los recuerdos, las expectativas y que exige el replanteamiento del estilo de vida, puede ser catalogado como todo un acontecimiento en el plano personal y familiar y como tal debe entenderse por los aquellos profesionales responsables de compartir esos instantes tan cargados de significado.

\section{V.- CONCLUSIONES}

Leyendo a Heidegger, especialmente su obra "El ser y el tiempo", se puede llegar a establecer el concepto fenomenológico de cuidado y el de cura. Paradójicamente, la enfermería, aun estando omnipresente ante el dolor, la angustia y la muerte, se ha mantenido cautelosamente alejada de la reflexión vivencial y fenomenológica del cuidado.

Las fuentes narrativas, concretamente una fábula escrita por Higinio en la primera mitad del siglo I a.C: "Fábula del hombre y el mundo", constituyen un referente esencial para entender la importancia filosófica, fenomenológica y epistemológica del cuidado. Esta fábula fue empleada por Heidegger para desarrollar su teoría fenomenológica.

- El tiempo constituye el cauce existencial por el que transcurren los diversos momentos históricos o "Kairós" que el ser humano (a nivel individual, familiar o comunitario) dedica a la satisfacción de todas y cada una de sus necesidades. El estudio del contexto temporal dedicado al proceso de satisfacción de necesidades incide tanto en la forma en la que se lleva a cabo dicho proceso como en su resultado.

- La fenomenología constituye un recurso metodológico de gran pertinencia para reflexionar sobre los aspectos éticos de los cuidados. 
-El cuidado, conceptual y semánticamente, tiene un gran potencial que pude contribuir a la vertebración de la disciplina enfermera.

\section{BIBLIOGRAFÍA}

- ANNELSS, M. (1996) Hermeneutic phenomenology: philosophical perspectives and current use in nursing research. J Adv Nurs, 23/4: 705-13.

- ARÓSTEGUI, J. (2001) La investigación histórica: teoría y método. Crítica, Barcelona.

- BISHOP, A.H; SCUDDER, J.R (1997) A phenomenological interpretation of holistic nursing. J Holist Nurs, 15/2: 103-11

- BLOCH, M. (1999) Historia e historiadores. Akal, Madrid.

- BRAUDEL, F. (2002) La historia y las ciencias sociales. Alianza, Madrid.

- BUENO, G. (1978) "Reliquias y relatos: construcción del concepto de historia fenoménica". El Basilisco I:5.

- BURKE, p. (2003) Formas de hacer historia. Alianza, Madrid.

- FEBVRE, L. (1999) El combate por la historia. Ariel, Barcelona.

- GÓMEZ HERAS, J.M. (1989) El a priori del mundo de la vida: fundamentación fenomenológica de una ética de la ciencia y de la técnica. Anthropos, Barcelona.

- HAMILTON, D. (1993) The idea of history and the history of teh ideas. Image Journal Nursing Scholarship, 25/I: 25-50.

- HEIDEGGER, M. (2000) El ser y el tiempo. Fondo de Cultura Económica, Madrid.

- HUSSERL, (1991) La crisis de las ciencias europeas y la fenomenología trascendental. Crítica, Barcelona.

- HUSSERL, E. (1994) Problemas fundamentales de la fenomenología. Alianza, Madrid.

- LÜDTKE, A. (1991) Sobre los conceptos de vida cotidiana, articulación de las necesidades y "conciencia proletaria". Revista de Historia Social, 10: 41-61.

- NOLTE, E. (1989) Heidegger. Política e historia en su vida y en su pensamiento. Tecnos, Madrid.

- NOZICK, R. (1990) Anarquía, Estado y Utopía. Fondo de Cultura Económica. México, D.F.
- PALEY, J. (1996) Intuition and expertise: comments on the Benner debate. J Adv Nurs, 23/4: 665-71.

- PALEY, J. (1997) Husserl, phenomenology and nursing. J Adv Nurs, 26/1:187-93.

- PEARCE, S; RICHARDSON, A. (1996) Fatigue in cancer: a phenomenological perspective. Eur J Cancer Care, 5:/2: 111-5 - POMIAN, K. (1990) El orden del tiempo. Júcar, Madrid.

- SCHAFF, J. (1974) Historia y verdad. Grijalbo, México.

- SERRANO, M. (2006) Sobre la templanza del alma. Relato de un enfermero agredido en las Urgencias. Sobre la templanza del alma. Relato de un enfermero agredido en las Urgencias. Arch Memoria 2006; 3(2). Disponible en <http://www.indexf.com/memoria/3/a20609.php>

- SILES, J. (1997) Epistemología y enfermería.: por una fundamentación científica y profesional de la disciplina. Enfermería Clínica, IV/7: 188-194.

- SILES, J. (1999) Historia de la enfermería. Aguaclara, Alicante.

- SILES, J. (2004) La construcción social de la historia de la enfermería. Index de enfermería. 13/ 47: 7-10.

- SILES, J. (2004) La construcción social de la historia de la enfermería. Index de enfermería. 13/ 47: 7-10.

- SILES, J. (2005) La eterna guerra de la identidad enfermera: un enfoque dialéctico y deconstruccionista- Index de enfermería. 14/50: 7-9.

- SILES, J. (2005) La eterna guerra de la identidad enfermera: un enfoque dialéctico y deconstruccionista- Index de enfermería. 14/50: 7-9.

- SOLANO, C; y SILES, J. (2005) Las vivencias del paciente coronario en la unidad de cuidados críticos. Index de Enfermería. 51: 29-33.

- SILES, J y SOLANO, C. (2007) Estructuras sociales, división sexual del trabajo y enfoques metodológicos. La estructura familiar y la función socio-sanitaria de la mujer. Invest Educ Enferm. 25/1: 66-73.

- VILAR, P. (2001) Crecimiento y desarrollo. Economía e historia. Reflexiones sobre el caso español Crítica, Barcelona.

- WHILTROW, G.H. (1990) El tiempo en la historia. La evolución de nuestro sentido del tiempo y de la perspectiva temporal. Crítica, Barcelona.

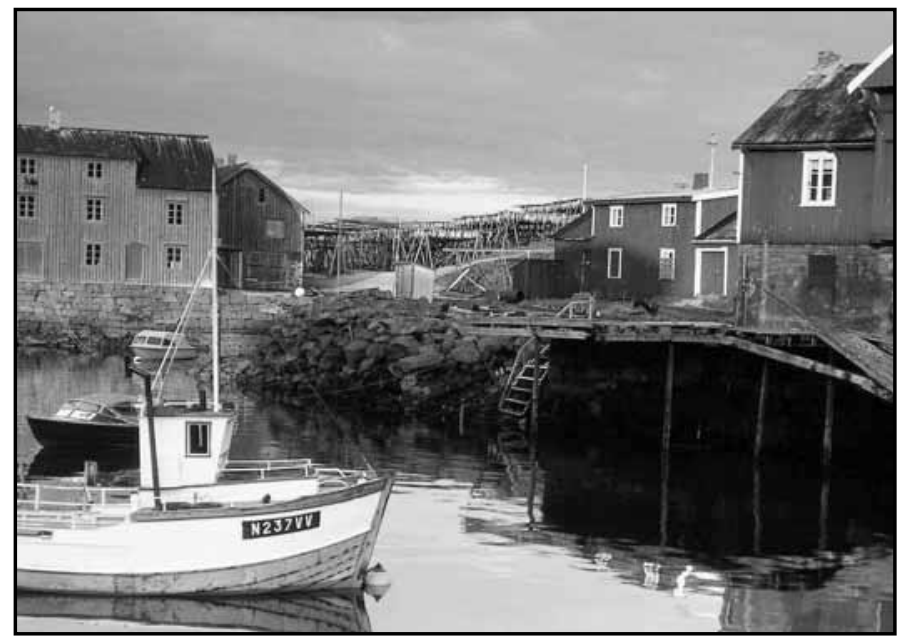

